\title{
ELEVATED SERUM LIVER-TYPE FATTY ACID BINDING PROTEIN LEVELS IN NON- ACETAMINOPHEN ACUTE LIVER FAILURE PATIENTS WITH ORGAN DYSFUNCTION.
}

\section{Karvellas, Constantine $\mathrm{J}^{1,5,6}$. and Speiser, Jaime $\mathrm{L}^{2}{ }^{2}$ and Tremblay, Mélanie $^{3}$ and Lee, William $\mathrm{M}^{4}$. and Rose, Christopher F. ${ }^{3,5}$ and Group, U.S. Acute Liver Failure Study}

* Constantine J. Karvellas

cjk2@ualberta.ca

Jaime L. Speiser

jspeiser@wakehealth.edu

Mélanie Tremblay

melanie.tremblay@ hepato $\square$ neuro.ca

1 Divisions of Hepatology and Critical Care Medicine, University of Alberta, Edmonton, Canada

2 Department of Biostatistics and Data Science, Wake Forest School of Medicine, Winston Salem, NC, USA

3 Hepato $\square$ neuro Laboratory, CRCHUM, Université de Montréal, Montreal, Canada

4 Division of Digestive and Liver Diseases, Department of Internal Medicine, University of Texas Southwestern Medical Center, Dallas, TX, USA

5 Canadian Donation and Transplant Research Program, Edmonton, Canada

\section{ABSTRACT}

Background Liver-type fatty acid binding protein (FABP1) has previously been demonstrated to improve prognostic discrimination in acetaminophen (APAP)-induced ALF but has not been investigated in other etiologies of ALF. Aim To determine whether FABP1 levels (early: admission or late: days 3-5) are associated with 21-day transplant-free survival in non-APAP ALF. Methods FABP1 was measured in serum samples from 384 ALF patients $(\mathrm{n}=88$ transplant-free survivors (TFS), n=296 died/LT-NTFS) using solid-phase enzyme-linked immunosorbent assay and analyzed with US ALFSG registry data. Results Of 384 ALF patients (autoimmune hepatitis n=125, drug-induced liver injury $n=141$, Hepatitis $B$ $\mathrm{n}=118), 177$ (46\%) patients received LT. Early FABP1 levels were significantly higher in ALF patients requiring vasopressor support (203.4 vs. $76.3 \mathrm{ng} / \mathrm{mL}$ ) and renal replacement therapy (203.4 vs. $78.8 \mathrm{ng} / \mathrm{mL} ; \mathrm{p}<0.001$ for both). Late FABP1 levels were significantly higher in patients requiring mechanical ventilation $(77.5 \mathrm{vs.} 53.3 \mathrm{ng} / \mathrm{mL})$, vasopressor support ( 116.4 vs. $53.3 \mathrm{ng} / \mathrm{mL}$ ) and in patients with grade $3 / 4$ hepatic encephalopathy $(71.4 \mathrm{vs.} 51.4 \mathrm{ng} / \mathrm{mL} ; \mathrm{p}=0.03$ for all). Late FABP1 levels were significantly lower in TFS patients (TFS 54 vs. NTFS $66 \mathrm{ng} / \mathrm{mL} ; \mathrm{p}=0.049$ ) but not admission (TFS 96 vs. NTFS $87 \mathrm{ng} / \mathrm{mL}$; $\mathrm{p}=0.67$ ). After adjusting for significant covariates, serum FABP1 did not discriminate significantly between TFS and patients who died/received LT at day 21 either on admission $(\mathrm{p}=0.29)$ or late (days $3-5, \mathrm{p}=0.087)$ time points. Conclusion In this first report of FABP1 in non-APAP ALF, FABP1 levels at late time points (days 3-5) were 
This is a post-peer-review, pre-copyedit version of an article published in Dig Dis Sci. The final authenticated version is available online at: http://dx.doi.org/10.1007/s10620-020-06166-w".

significantly lower in ALF patients who were alive without transplant at day 21 but not after adjusting for covariates reflecting severity of illness. Higher FABP1 levels were associated with the presence of increased organ failure.

Keywords Acute liver failure $\cdot$ Liver-type fatty acid binding protein $\cdot$ Multiorgan failure $\cdot$ Prognosis $\cdot$ ALFSG index

\section{ALF Acute liver failure}

ALFSG Acute Liver Failure Study Group

APAP Acetaminophen

FABP1 Liver-type fatty acid binding protein

HE Hepatic encephalopathy

ICU Intensive Care Unit

INR International normalized ratio

IQR Interquartile range

\section{ABbreviations}

$\begin{array}{ll}\text { KCC } & \text { King's College Criteria } \\ \text { LT } & \text { Liver transplantation } \\ \text { MAP } & \text { Mean arterial pressure }(\mathrm{mm} \mathrm{Hg}) \\ \text { MELD } & \text { Model for end-stage liver disease score } \\ \text { MV } & \text { Mechanical ventilation } \\ \text { OR } & \text { Odds ratio } \\ \text { RRT } & \text { Renal replacement therapy } \\ \text { TFS } & \text { Transplant-free survivor at day } 21\end{array}$

\section{INTRODUCTION}

Acute liver failure (ALF) is defined by the occurrence of encephalopathy and hepatic synthetic dysfunction within 26 weeks of the first symptoms of liver disease [1]. In many previous studies, patients have been divided with ALF between those due to acetaminophen (APAP) and those with other etiologies since the groups vary greatly in outcomes. Criteria for predicting outcome such as the Kings College Criteria (KCC) initially published in 1989 when utilized in further studies have shown relatively poor sensitivity of the non-APAP criteria, ranging between 60 and $70 \%$ [2]. More recently, the ALFSG prognostic index also demonstrated improved accuracy (c-statistic 0.84) but limited sensitivity (37\%) in ALF [3].

Liver transplant (LT) in ALF presents significant challenges due to the rapidity and severity of illness (i.e., high risk of cerebral edema and multiorgan failure), the potential for recovery without LT, and the presence of concomitant complex psychosocial issues (contraindications) [4]. Given changes in critical care management of ALF, some patients may have a good outcome despite meeting poor prognostic criteria and potentially could avoid unnecessary LT in the presence of improved prognostic markers or scores [5].

Fatty acid binding proteins (FABP) are small $(<15 \mathrm{kDa})$ cytoplasmic proteins that are abundantly expressed in tissues with active fatty acid metabolism, including hepatocytes. The primary function of FABPs is the intracellular transport of long-chain fatty acids [6]. The cellular expression of FABPs is responsive to changes in lipid metabolism, which can be induced by pathophysiological conditions, such as ischemia/inflammation [7]. Liver-type FABP (FABP1), significantly expressed in hepatocytes, enterocytes, and to a lesser degree in renal tubular cells, has been associated with liver injury [6]. Recently, we evaluated the discriminatory ability of FABP1 in 198 APAP ALF patients [8]. Elevated FABP1 levels were associated with significantly increased mortality and improved prognostic discrimination when added to KCC and the ALFSG prognostic index [8]. However, to date FABP1 has never been evaluated in other causes of ALF.

This nested case-control study of randomly selected samples from prospectively enrolled patients from the US Acute Liver Failure Study Group (ALFSG) registry aimed to examine levels of FABP1 in non-APAP ALF patients. Specifically, our primary objectives were to test the following hypotheses:

Karvellas, C. J.; Speiser, J. L.; Tremblay, M.; Lee, W. M.; Rose, C. F. \& Group, U. A. L. F. S. Elevated Serum Liver-Type Fatty Acid Binding Protein Levels in Non-acetaminophen Acute Liver Failure Patients with Organ Dysfunction. Digest Dis Sci, 2020 
This is a post-peer-review, pre-copyedit version of an article published in Dig Dis Sci. The final authenticated version is available online at: http://dx.doi.org/10.1007/s10620-020-06166-w".

(a) Higher FABP1 serum levels are significantly associated with 21-day transplant-free mortality (in the absence of transplant) in non-APAP ALF patients.

(b) Elevated serum levels of FABP1 in non-APAP ALF are significantly associated with 21-day transplant-free mortality after adjusting for other significant covariates.

\section{Methods}

\section{STUDY DESIGN}

This study is a nested case-control study of prospectively collected data and biosamples of 384 patients enrolled in the US ALFSG registry/biorepository and is outlined in detail in Supplementary File 1. Between January 1998 and December 2016, all patients with ALF due to autoimmune hepatitis $(\mathrm{AIH}, \mathrm{n}=125)$ and Hepatitis $\mathrm{B}(\mathrm{HBV}, \mathrm{n}=118)$ who had serial samples available (at least two sample collections from admission and at least $48 \mathrm{~h}$ later) were included in this analysis. Of 304 patients with ALF due to idiosyncratic (non-APAP) drug-induced liver injury (DILI) with serial samples, $n=141$ patients were selected at random by personnel not involved in the analysis of the samples or statistical analysis for the paper. All enrolling centers were tertiary academic centers and all but one were liver transplant centers. The authors' Institutional Review Board (IRB)/Health Research Ethics Boards of all enrolling US ALFSG sites have approved all research, and all clinical investigation has been conducted according to the principles expressed in the 1975 Declaration of Helsinki. Given patients were unable to provide written consent (critical illness, hepatic encephalopathy HE), written assent was obtained from the next of kin from each patient. Each center implemented monitoring and therapeutic interventions according to institutional standards of care. Reporting of the analysis of this study followed the STROBE Guidelines for reporting casecontrol studies [9]. Consistent with ALFSG studies [3], the primary outcome was 21-day transplant-free survival

\section{PARTICIPANTS}

Inclusion criteria were (1) evidence of ALF according to the enrollment criteria for the ALFSG (see operational definitions) and 2) age $\geq 18$ years; (2) HE during the first seven days of study admission (West Haven Criteria) [10, 11]; and (3) patients within the ALFSG registry with primary diagnoses of Hepatitis B (HBV), autoimmune (AIH), and idiosyncratic (nonAPAP) drug-induced liver injury (DILI) determined by the site investigator. Exclusion criteria were: (1) cirrhosis/acute-onchronic liver failure and (2) patients with a primary diagnosis of APAP. Serum samples were analyzed on study admission early (day 1) and late (either days 3, 4, or 5 where available). Patients who received a LT were included in this study and were considered as an unfavorable outcome, in keeping with other previous studies.

\section{OPERATIONAL DEFINITIONS}

For the purposes of this study, ALF was defined as INR $\geq 1.5$ and HE within the first 26 weeks of liver disease in a patient with an acute hepatic insult [1]. HE grade was defined by the West Haven Criteria (simplified) as follows: grade 1 any alteration in mentation, grade 2 being somnolent or obtunded but easily rousable or presence of asterixis, grade 3 being rousable with difficulty, and grade 4: unresponsive to deep pain $[10,11]$. In this study, we defined "low coma grade" as grade 1 or 2 and "high coma grade" as grade 3 or 4. The KCC [12] predicts poor outcome (death/transplant) if (a) INR is greater than 6.5 or three of the following (b) INR is greater than 3.5, bilirubin is greater than $17 \mathrm{mg} / \mathrm{dL}$, age less than 10 or greater than 40 years, jaundice for greater than 7 days before development of encephalopathy or unfavorable etiology (DILI). The model for end-stage liver disease (MELD) is defined as $10 *(0.957 * \log (4)+0.378 * \log$ (bilirubin) $+1.12 * \log ($ INR $)$ ) for dialyzed patients and $10 *(0.957 * \log ($ creatinine $)+0.378 * \log ($ bilirubin $)+1.12 * \log ($ INR $))$ for patients not dialyzed [13]. For low coma grade, HE insert 0 , and for high coma grade, insert 1 . HBV, AIH and DILI are defined by the ALFSG index as unfavorable etiologies (insert 0). For the absence of vasopressor use, insert 0 , for vasopressor use, insert 1.

Karvellas, C. J.; Speiser, J. L.; Tremblay, M.; Lee, W. M.; Rose, C. F. \& Group, U. A. L. F. S. Elevated Serum Liver-Type Fatty Acid Binding Protein Levels in Non-acetaminophen Acute Liver Failure Patients with Organ Dysfunction. Digest Dis Sci, 2020 
This is a post-peer-review, pre-copyedit version of an article published in Dig Dis Sci. The final authenticated version is available online at: http://dx.doi.org/10.1007/s10620-020-06166-w".

LABORATORY ASSAYS OF FABP1

FABP1 was measured in serum samples with a solid-phase enzyme-linked immunosorbent assay (ELISA) following manufacturer's instructions (Biomatik, USA). Briefly, samples were incubated $2 \mathrm{~h}$ on a monoclonal anti-FABP1 precoated plate. A specific FABP1 biotin-conjugated polyclonal antibody solution was added for $2 \mathrm{~h}$. After washing plates, avidin conjugated to horseradish peroxidase was added for $30 \mathrm{~min}$. Finally, substrate tetramethylbenzidine was added for $15 \mathrm{~min}$. Reactions were stopped by addition of sulfuric acid and absorbance was read at $450 \mathrm{~nm}$. Standard curve ranges from 1.56 to $200 \mathrm{ng} / \mathrm{mL}$. Samples were performed in duplicate and accepted valid with a variation coefficient less than $25 \%$. The model used for the ELISA standard curves was the fourparameter logistic (4-PL) curve fit, with all standard R2 curves superior to 0.999 using Graph Pad Prism (Sand Diego, USA).

\section{CLINICAL VARIABLES}

Clinical, biochemical and outcome data were collected prospectively as part of the US ALFSG registry to be analyzed for the 384 patients in this study. Data assessed in this study from the registry included demographic (age, race, sex), comorbidities, biochemistry early and late (complete blood count, creatinine, transaminases, phosphate, international normalized ratio (INR), bilirubin, ammonia, lactate), hepatic coma grade, and requirement for organ support (mechanical ventilation (MV), vasopressors, renal replacement therapy (RRT)).

\section{STATISTICAL METHODS}

For differences between outcome groups (ALF transplant-free survivors, $n=88$, ALF non-transplant-free survivors, $n=296$ ), categorical variables were compared using the Chi-squared test or Fisher's exact test (if $n<10$ in any cell of the two-bytwo table). FABP1 was treated as a continuous variable. Continuous variables were reported as medians with interquartile range (IQR) and compared using the Wilcoxon rank sum test. Transplant-free survival was defined as the dichotomous outcome, alive or dead/LT at 21 days after enrollment into the registry. A two-sided $p$ value of $<0.05$ was considered statistically significant for all comparisons. In order to control for variables that may confound the effect of FABP1 on 21-day mortality, logistic regression analysis was performed [14]. Aside from FABP1, covariates considered in multivariable modeling included MELD, lactate, vasopressors use, RRT, MV, and high coma grade. Separate multivariable (logistic) regression models were derived for FABP1 early (day 1) and late (days 3-5) by performing backward elimination with a $\mathrm{p}$ value threshold of 0.05. Model performance was assessed using area under the receiver operating curve (AUROC) and the Hosmer-Lemeshow test for goodness of fit. SAS software version 9.4 was used for univariate comparisons and multivariable logistic regression modeling.

\section{RESULTS}

COMPARATIVE ANALYSIS OF 384 ALF PATIENTS

Demographic and clinical outcomes stratified by transplant-free survival (alive at day 21, n=88; deceased/LT, n=296) are shown in Table 1.

Comparing ALF TFS and deceased/LT at day 21, there were no significant differences in age ( 45 vs. 47, p=0.39) or gender (female; $66 \%$ vs. $66 \%, \mathrm{p}=0.93$ ). ALF TFS required significantly less organ support during the 7 days of inpatient study (MV: $21 \%$ vs. $66 \%$ and vasopressors $10 \%$ vs. $35 \%$; $\mathrm{p}<0.0001$ for both). ALF TFS patients were less likely to achieve high (3 or 4) HE coma grade (30\% vs. 73\%, p < 0.0001). ALF TFS patients were less likely to require blood products during the first 7 days of study including red blood cells (5\% vs. 12\%, p<0.046), fresh frozen plasma (15\% vs. 44\%, $\mathrm{p}<0.0001$ ), and platelets ( $1 \%$ vs. $8 \%, \mathrm{p}=0.014$ ). On admission, $46 \%$ of ALF TFS and $69 \%$ of ALF non-survivors/LT patients met $\mathrm{KCC}(\mathrm{p}=0.0001)$.

Karvellas, C. J.; Speiser, J. L.; Tremblay, M.; Lee, W. M.; Rose, C. F. \& Group, U. A. L. F. S. Elevated Serum Liver-Type Fatty Acid Binding Protein Levels in Non-acetaminophen Acute Liver Failure Patients with Organ Dysfunction. Digest Dis Sci, 2020 
This is a post-peer-review, pre-copyedit version of an article published in Dig Dis Sci. The final authenticated version is available online at: http://dx.doi.org/10.1007/s10620-020-06166-w".

Table 1 Overall. Demographic and Clinical Parameters in 384 ALF patients stratified by Day 21 outcome.

\begin{tabular}{|c|c|c|c|c|c|}
\hline & \multicolumn{2}{|r|}{$\begin{array}{l}\text { Alive Day } 21 \\
(n=88)\end{array}$} & \multicolumn{2}{|r|}{$\begin{array}{l}\text { LT/Dead Day } 21 \\
\qquad(n=296)\end{array}$} & \multirow[b]{2}{*}{ Pvalue } \\
\hline & $\mathrm{N}$ & $\begin{array}{c}\text { Number }(\%) \text { or median } \\
\text { (IOR) }\end{array}$ & $\mathrm{N}$ & $\begin{array}{c}\text { Number }(\%) \text { or median } \\
\text { (IOR) }\end{array}$ & \\
\hline Age & 88 & $45(32-56)$ & 296 & $47\{35-58\}$ & 0.39 \\
\hline Sex (female) & 88 & $58(65.9)$ & 296 & $196\{66.2\rangle$ & 0.96 \\
\hline \multicolumn{6}{|l|}{ Race } \\
\hline White & 88 & $53\langle 60.2\rangle$ & 298 & $179\{60.5\rangle$ & \multirow{3}{*}{0.20} \\
\hline African-American & 88 & $27(30.7)$ & 296 & $71(24.0)$ & \\
\hline Other & 88 & $8(9.1)$ & 296 & $46(15.5)$ & \\
\hline \multicolumn{6}{|l|}{ Organ support (days 1-7) } \\
\hline Mechanical ventilation & 88 & $18(20.5)$ & 296 & $194(65.5)$ & $\$ 0.0001$ \\
\hline Vasopressors & 88 & $9(10.2)$ & 296 & $103(34.8)$ & $\infty 0.0001$ \\
\hline Renal Replaciement therapy & 88 & $11(12.5)$ & 296 & $49(16.6)$ & 0.36 \\
\hline $\mathrm{KCC}$ & 82 & $38(46.3)$ & 286 & $198(69.2)$ & 0.0001 \\
\hline Coma Grade $3 / 4$ (Worst days 1-7) & 87 & $26(29.9)$ & 291 & $211(72.5)$ & 40.0001 \\
\hline \multicolumn{6}{|l|}{ ICP directed therapies (days 1-7) } \\
\hline ICP Monitor & 80 & $4(5.0)$ & 248 & $25(10.1)$ & 0.26 \\
\hline Mannitol & 88 & $0(0)$ & 294 & $34(11.6)$ & 0.066 \\
\hline Hypertonic saline & 88 & $3(3.4)$ & 293 & $12(4.1)$ & 1.0 \\
\hline Barbiturates & 88 & $2(2.3)$ & 294 & $7(2.4)$ & 1.0 \\
\hline Hypothermia & 88 & $3(3.4)$ & 293 & $6(2.1)$ & 0.44 \\
\hline $\begin{array}{l}\text { Sedatives } \\
\text { Sed }\end{array}$ & 88 & $17(19.3)$ & 294 & $102(34.7)$ & 0.0063 \\
\hline \multicolumn{6}{|l|}{ Blood products (days 1-7) } \\
\hline Red Biood Cells & 88 & $4(4.6)$ & 294 & $36(12.2)$ & 0.046 \\
\hline Fresh Frozen Plasma & 88 & $13(14.8)$ & 294 & $130(44.2)$ & $<0.0001$ \\
\hline Recombinant VIIA & 88 & $0(0)$ & 293 & $1(0.3)$ & 1.0 \\
\hline Platelets & 88 & $1\{1.1)$ & 294 & $24\langle 8.2\rangle$ & 0.014 \\
\hline \multicolumn{6}{|l|}{ ICU Complications (days 1-7) } \\
\hline Seizures & 88 & $1(1.1)$ & 293 & $5(1.7)$ & 1.0 \\
\hline Arrhythmias & 88 & $6(6.8)$ & 294 & $32\{10.9\}$ & 0.26 \\
\hline Gl bleeding & 88 & $0\langle 0\rangle$ & 294 & $11(3.7)$ & 0.075 \\
\hline Abnormal CT & 8 & $6(75.0)$ & 59 & $51\langle 86.4\rangle$ & 0.34 \\
\hline Abnormal CXR & 57 & $18(31.6)$ & 226 & $94(41.6)$ & 0.17 \\
\hline Backeremia/Blood stream infection & 88 & $5(5.7)$ & 293 & $14(4.8)$ & 0.73 \\
\hline Died by day 21 & 88 & $0(0)$ & 296 & $134(45.3)$ & $\$ 0.0001$ \\
\hline \multicolumn{6}{|l|}{ Cause of death } \\
\hline Multicrgan fallure & & & 134 & $104(77.6)$ & \\
\hline Cerebral edema & & & 134 & $20(14.9)$ & \\
\hline Unknown & & & 134 & $10(7.5)$ & \\
\hline \multicolumn{6}{|l|}{ LT } \\
\hline Listed & 87 & $15(17.2)$ & 292 & $212(72.6)$ & $\propto 0.0001$ \\
\hline Received & 87 & $0(0)$ & 293 & $177\langle 60.4\rangle$ & $<0.0001$ \\
\hline \multicolumn{6}{|l|}{ Etiology } \\
\hline Auboimmune hepatits & 88 & $24(27.3)$ & 296 & $101(34.1)$ & \multirow{3}{*}{0.31} \\
\hline DILI & 88 & $38(43.2)$ & 296 & $103(34.8)$ & \\
\hline Hepastis B & 88 & $26(29.6)$ & 296 & $92(31.1)$ & \\
\hline
\end{tabular}

$\mathrm{N}$ : frequency. IQR: interquartile range.

$\mathrm{CT}$ : computed tomography. CXR; chest x-ray.

Among the 134 ALF non-survivors (irrespective of LT), the most common causes of death reported were multiorgan failure (73\%) and neurological complications (15\%). Cause of death was unknown in $8 \%$ of cases.

Data were stratified by etiology of ALF (AIH, HBV, DILI) and are shown in Supplementary Tables 1, 3, and 5.

\section{CLINICAL PARAMETERS IN 384 ALF PATIENTS: AdMISSION (EARLY)}

Comparisons of clinical parameters by outcome group on study admission are shown in Table 2. ALF TFS survivors had significantly lower serum INR (2.1 vs. 3.2) and bilirubin (16.6 vs. $22.1 \mathrm{mg} / \mathrm{dl}$ ) compared to ALF LT/nonsurvivors ( $\mathrm{p}<0.0001$ for all comparisons). Transplant-free survivors also demonstrated significantly lower MELD scores (20 vs. $27, \mathrm{p}<0.0001$ ) than ALF LT/non-survivors on admission. On study admission, survivors were significantly less likely to be mechanically ventilated ( $14 \%$ vs. $43 \%$, p<0.0001) or achieve high HE grade $(21 \%$ vs. $48 \%$, p<0.0001).

Karvellas, C. J.; Speiser, J. L.; Tremblay, M.; Lee, W. M.; Rose, C. F. \& Group, U. A. L. F. S. Elevated Serum Liver-Type Fatty Acid Binding Protein Levels in Non-acetaminophen Acute Liver Failure Patients with Organ Dysfunction. Digest Dis Sci, 2020 
This is a post-peer-review, pre-copyedit version of an article published in Dig Dis Sci. The final authenticated version is available online at: http://dx.doi.org/10.1007/s10620-020-06166-w".

Admission (early) levels of FABP1 are shown in Table 2 and graphically in Fig. 1. There were no statistically significant differences in FABP1 levels between ALF TFS (96.3 vs. $87.2 \mathrm{ng} / \mathrm{mL}$ ) and ALF patients that died/required LT $(\mathrm{p}=0.67)$. Data stratified by etiology of ALF (AIH, HBV, and DILI) are shown in Supplementary Tables 2, 4, and 6.

\section{CLINICAL PARAMETERS IN 328 ALF PATIENTS: DAY 3-5 (LATE)}

Comparisons of clinical parameters by outcome group on days 3-5 (late) are shown in Table 2. Of the 88 ALF TFS, 78 patients had data available at a late time point. Of the 296 ALF patients who died/required LT, 250 patients had data at a late time point. At late time points, ALF TFS patients $(\mathrm{n}=78)$ had significantly lower serum INR (2.0 vs. 2.8), bilirubin ( 17.3 vs. $20.6 \mathrm{mg} / \mathrm{dl}$ ), and lactate levels ( 2.2 vs. $4.7 \mathrm{mmol} / \mathrm{L}$ ) compared to ALF non-survivors/LT recipients $(\mathrm{n}=250 ; \mathrm{p}<0.02$ for all comparisons). ALF TFS patients also demonstrated significantly lower late MELD scores (19 vs. 27, $\mathrm{p}<0.0001)$ than non-survivors. At late time points, ALF TFS patients were significantly less likely to be on mechanical ventilation ( $15 \%$ vs. $51 \%)$ and vasopressors ( $4 \%$ vs. $23 \%)$ or achieve high $\mathrm{HE}$ grade $(20 \%$ vs. $56 \%$, p $<0.0001$ for all comparisons).

Late (days 3-5) levels of FABP1 are shown in Table 2 and graphically in Fig. 1. ALF TFS patients had significantly lower late serum FABP1 levels (53.8 vs. $65.6 \mathrm{ng} / \mathrm{mL}$ ) compared with ALF non-survivors/LT recipients $(\mathrm{p}=0.049)$. Data stratified by etiology of ALF (AIH, HBV, and DILI) are shown in Supplementary Tables 2, 4, and 6.

\section{CORRELATION BETWEEN FABP1 LEVELS AND ORGAN DYSFUNCTION/REQUIREMENT FOR SUPPORT}

ALF patients were stratified based on requirements for organ support in Table 3. At early time points, serum FABP1 levels were significantly higher in ALF patients requiring vasopressor support (203.4 vs. $76.3 \mathrm{ng} / \mathrm{mL})$ and renal replacement therapy ( 203.4 vs. $78.8 \mathrm{ng} / \mathrm{mL}$; $<<0.001$ for both). At late time points, serum FABP1 levels were significantly higher in ALF patients requiring mechanical ventilation ( $77.5 \mathrm{vs.} 53.3 \mathrm{ng} / \mathrm{mL}, \mathrm{p}=0.006$ ), vasopressor support (116.4 vs. $53.3 \mathrm{ng} / \mathrm{mL} ; \mathrm{p}<0.001$ ) and in ALF patients with high-grade hepatic encephalopathy (grade 3 or $4 ; 71.4 \mathrm{vs} .51 .4 \mathrm{ng} / \mathrm{mL}$; $\mathrm{p}=0.03)$.

MULTIVARIABLE ANALYSIS: ASSOCIATIONS WITH 21DAY MORTALITY

In order to adjust for covariates, multivariable logistic regression for 384 ALF patients to determine associations (adjusted) with 21-day mortality was performed (Table 3). Two models were derived; one on admission (early) and one at days 3-5 (late). Values of serum FABP1 were transformed to their natural logarithm (log FABP1) to comply with the linearity assumption in logistic regression.

EARLY (ADMISSION) MODEL

After adjusting for covariates, the multivariable early model included requirement for high coma grade (odds ratio OR 3.97, 95\% CI (2.01-7.85), p<0.0019), $\log$ (INR) (OR $5.38(2.75,10.53), \mathrm{p}<0.0001)$ and bilirubin (OR $1.10(1.06,1.14)$ per increment, $\mathrm{p}<0.0001$ ), and etiology of ALF ( $\mathrm{p}=0.27)$. Log (FABP1) (OR 1.13 (0.90-1.40) per unit, $\mathrm{p}=0.29)$ was not independently associated with death/LT after adjusting for other covariates. This model demonstrated AUROC of 0.82. While etiology of ALF was not statistically significant ( $\mathrm{p}=0.27$ ), early multivariable models stratified by etiology of ALF (AIH, HBV, and DILI) are shown in Supplementary Tables 7, 8, and 9.

\section{LATE (DAYS 3-5)}

Model After adjusting for covariates, the multivariable late (days 3-5) model included MELD (OR 1.09 (1.051.14) per increment, $\mathrm{p}<0.0001$ ) and etiology of ALF ( $\mathrm{p}=0.67)$. Log (FABP1) (OR $1.27(0.97-1.68)$ per unit, $\mathrm{p}=0.087$ ) was not independently associated with death/LT after adjusting for other covariates. This model demonstrated AUROC of 0.76 .

Karvellas, C. J.; Speiser, J. L.; Tremblay, M.; Lee, W. M.; Rose, C. F. \& Group, U. A. L. F. S. Elevated Serum Liver-Type Fatty Acid Binding Protein Levels in Non-acetaminophen Acute Liver Failure Patients with Organ Dysfunction. Digest Dis Sci, 2020 
This is a post-peer-review, pre-copyedit version of an article published in Dig Dis Sci. The final authenticated version is available online at: http://dx.doi.org/10.1007/s10620-020-06166-w".

While etiology of ALF was not statistically significant $(\mathrm{p}=0.67)$, late multivariable models stratified by etiology of ALF (AIH, HBV, and DILI) are shown in Supplementary Tables 7, 8, and 9.

A comparison of model performance (AUROC) for KCC, MELD, and the ALFSG prognostic index alone and with the addition of FABP1 is shown in Supplementary Table 10. Addition of FABP1 to KCC or MELD was not significant for early or late time points, nor was it significant when added to ALFSG PI for early time points; however, for late measures (days 3-7), addition of FABP1 to the ALFSG PI resulted in significant improvement in AUROC ( $\mathrm{p}=0.027)$.

Table 2 Overall. Biochemical and organ support parameters early (admission) and late (day 3-5) stratified 21-day outcome

Table 2 Overall. Biochemical and organ support parameters early (admission) and late (day 3-5) stratified 21day outcome.

\begin{tabular}{|c|c|c|c|c|c|}
\hline \multirow[t]{2}{*}{ EARLY $(n=384)$} & \multicolumn{2}{|r|}{ Alive Day $21(n=88)$} & \multicolumn{2}{|c|}{ LT/Dead Day $21(n=296)$} & \multirow[b]{2}{*}{ Pvalue } \\
\hline & $\mathrm{N}$ & $\begin{array}{c}\text { Number }(\%) \text { or median } \\
\text { (IOR) }\end{array}$ & $\mathrm{N}$ & $\begin{array}{c}\text { Number }(\%) \text { or median } \\
\text { (IOR) }\end{array}$ & \\
\hline \multicolumn{6}{|l|}{ Biochemistry } \\
\hline Hemoglobin (g/di) & 85 & $11.4(9.8-12.9)$ & 291 & $11.3(9.9-12.6)$ & 0.49 \\
\hline White Blood count $\left(10^{2} \Omega\right)$ & 84 & $9.2(5.9-12.6)$ & 292 & $102\{7.2-14.1\}$ & 0.048 \\
\hline Platelet count $\left(10^{2} / \mathrm{L}\right)$ & 85 & $153(110-212)$ & 290 & $132(93-192)$ & 0.025 \\
\hline INR & 82 & $2.1(1.7-2.7)$ & 283 & $3.2(2.3-4.8)$ & $<0.0001$ \\
\hline ALT (IUL) & 85 & $906(332-1782)$ & 282 & $579(255-1422)$ & 0.027 \\
\hline B lirubin (mgrdl) & 86 & $16.6(8.7-22.4)$ & 283 & $22.1(15.9-27.7)$ & $\infty .0001$ \\
\hline $\mathrm{pH}$ & 32 & $7.45(7.38-7.52)$ & 184 & $7.46(7.40-7.51)$ & 0.65 \\
\hline Ammonia (venous) (umol/L) & 25 & $73(48-106)$ & 97 & $104(78-168)$ & 0.0011 \\
\hline Creatinine (mg/dL) & 86 & $1.1(0.7-2.2)$ & 289 & $1.1(0.7-2.1)$ & 0.61 \\
\hline Lactate (mmo/li) & 26 & $3.4(1.8-6.6)$ & 103 & $4.8(2.7-7.6)$ & 0.066 \\
\hline Phosphate (mg/dL) & 53 & $2.9(2.1-3.7)$ & 200 & $3.4(2.4-4.7)$ & 0.025 \\
\hline MELD & 80 & $20(16-26)$ & 273 & $27(21-34)$ & 40.0001 \\
\hline $\begin{array}{l}\text { High Coma Grade ( } 3 \text { or } \\
4)^{*}\end{array}$ & 86 & $18(20.9)$ & 287 & $138(48.1\rangle$ & $\varnothing 0.0001$ \\
\hline \multicolumn{6}{|l|}{ Organ support } \\
\hline Mechanical ventlation & 88 & $12(13.6)$ & 294 & $125(42.5)$ & $\infty 0.0001$ \\
\hline Vasopressors & 88 & $7(8.0)$ & 294 & $41(14.0)$ & 0.14 \\
\hline $\begin{array}{r}\text { Renal Replacement } \\
\text { therapy }\end{array}$ & 88 & $5(5.7)$ & 294 & $32(10.9)$ & 0.15 \\
\hline FABP1 (ngimi) & 88 & $96.3\{41.8-240.4\}$ & 288 & $872(38.9-223.1\}$ & 0.67 \\
\hline \multirow[t]{2}{*}{ LATE $(n=328)$} & \multicolumn{2}{|r|}{ Alive Day $21(n=78)$} & \multicolumn{2}{|c|}{ LT/Dead Day $21(n=250)$} & \\
\hline & $\mathrm{N}$ & $\begin{array}{c}\text { Number }(\%) \text { or median } \\
\text { (IOR) }\end{array}$ & $\mathrm{N}$ & $\begin{array}{c}\text { Number (\%) or median } \\
\text { (IOR) }\end{array}$ & Pvalue \\
\hline \multicolumn{6}{|l|}{ Biochemistry } \\
\hline Hemoglobin (g/dL) & 71 & $11.3(9.7-12.5)$ & 228 & $10.6\{9.4-12.3\}$ & 0.11 \\
\hline White Blood count $\left(10^{9} / \mathrm{L}\right)$ & 71 & $9.1(6.5-14.5)$ & 228 & $9.9(7.1-14.5)$ & 0.58 \\
\hline Platelet count $\left(10^{2} / \mathrm{L}\right)$ & 70 & $151(89-190)$ & 227 & $108(63-159)$ & 0.0007 \\
\hline INR & 72 & $2.0(1.6-2.5)$ & 217 & $2.8(2.0-4.1)$ & $<0.0001$ \\
\hline ALT (IUI) & 74 & $670(269-1139)$ & 224 & $468(218-940)$ & 0.18 \\
\hline Bilirubin (mg/dL) & 71 & $17.3(9.5-23.7)$ & 223 & $20.6(14.6-272)$ & 0.012 \\
\hline $\mathrm{pH}$ & 18 & $4.46(7.42-7.50)$ & 156 & $7.44(7.39-7.50)$ & 0.38 \\
\hline Ammonia (venous) (umolili) & 17 & $64(54-86)$ & 57 & $102(87-122)$ & 0.0008 \\
\hline Creatinine (mg/dL) & 73 & $0.9\langle 0.7-1.5\rangle$ & 231 & $1.1(0.8-2.1)$ & 0.13 \\
\hline Lactate (mmolli) & 15 & $2.2(1.7-3.3)$ & 67 & $4.7(2.8-8.6)$ & 0.0004 \\
\hline Phosphate (mg/dL) & 36 & $3.0\{2.6-4.1\}$ & 101 & $3.3\{2.7-4.6\}$ & 0.34 \\
\hline MELD & 65 & $19\{15-23\}$ & 205 & $27(20-33)$ & $\varnothing 0.0001$ \\
\hline $\begin{array}{l}\text { High Coma Grade ( } 3 \text { or } \\
4)^{*}\end{array}$ & 71 & $14(19.7)$ & 237 & $133(56.1)$ & $\infty 0.0001$ \\
\hline \multicolumn{6}{|l|}{ Organ support } \\
\hline Mechanical ventlation & 78 & $12(15.4)$ & 245 & $124(50.6)$ & $\infty 0.0001$ \\
\hline Vasopressors & 78 & $3\{3.9\}$ & 245 & $57(23.3)$ & $<0.0001$ \\
\hline $\begin{array}{r}\text { Renal Replacement } \\
\text { therapy }\end{array}$ & 78 & $4(5.1)$ & 245 & $27(11.0)$ & 0.18 \\
\hline FABP1 (ng/mi) & 78 & $53.8(28.9-128.4)$ & 237 & $65.6(35.3-195.1)$ & 0.049 \\
\hline
\end{tabular}

$\mathrm{N}$, frequency; IQR, interquartile range; INR, international normalized ratio; ALT, alanine aminotransferase; and MELD, model for end-stage liver disease

a Hepatic encephalopathy grade according to West Haven Criteria

Karvellas, C. J.; Speiser, J. L.; Tremblay, M.; Lee, W. M.; Rose, C. F. \& Group, U. A. L. F. S. Elevated Serum Liver-Type Fatty Acid Binding Protein Levels in Non-acetaminophen Acute Liver Failure Patients with Organ Dysfunction. Digest Dis Sci, 2020 


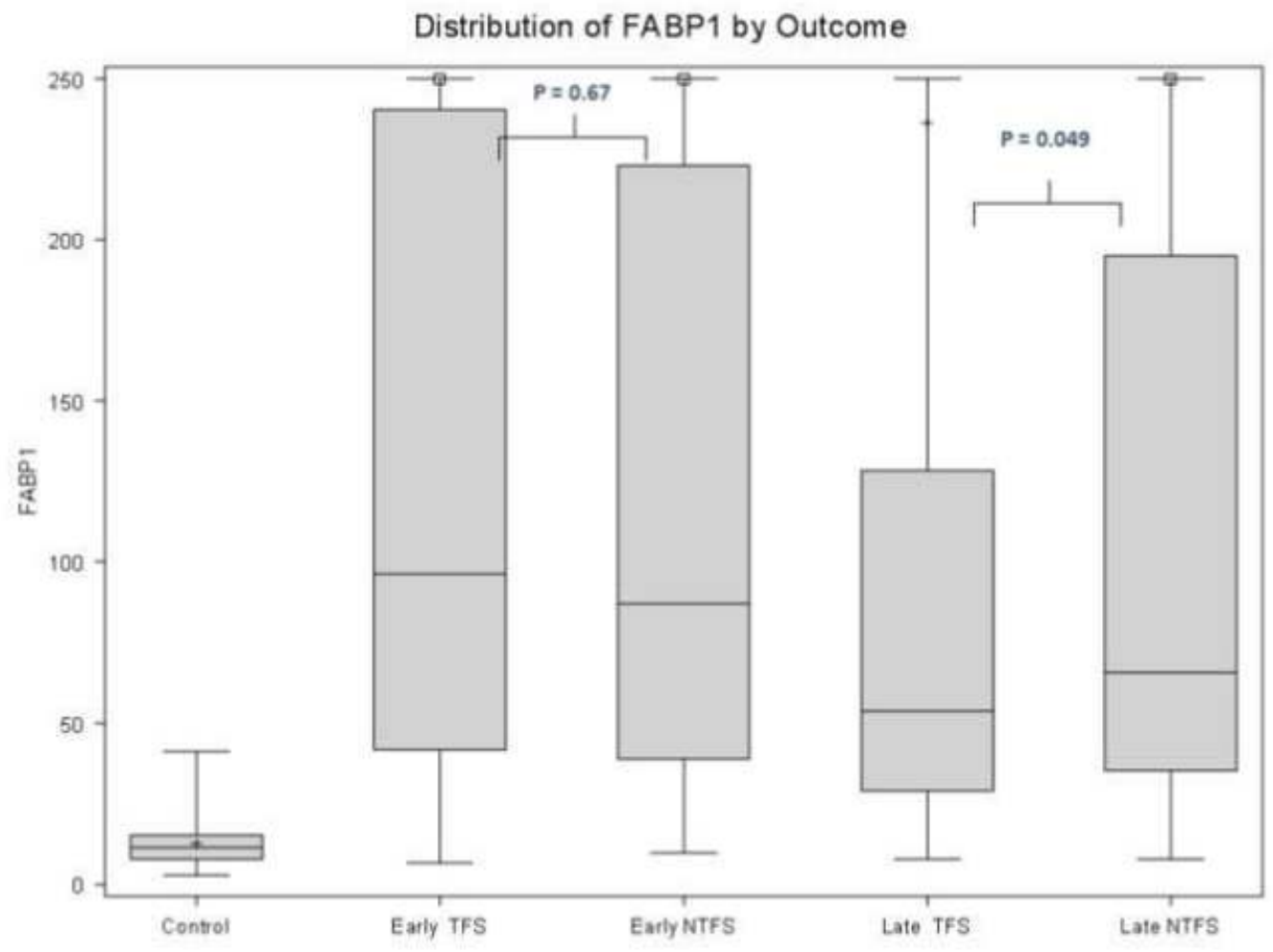

Fig. 1 Serum levels of FABP1 (ng/mL) in healthy controls, transplant-free survivors (TFS) (early admission), patients who died/required LT (NTFS, early), TFS (late day 3-5), NTFS (late)

Table 3 FABP1 median (IQR) for early and late samples by clinical descriptors

\begin{tabular}{lrlllc}
\hline & $N$ & Yes & $N$ & No & $p$ value \\
\hline Early FABP1 (ng/mL; median(IQR)) & & & & & \\
$\quad$ Mechanical ventilation & 132 & $118.5(40.1-284.7)$ & 242 & $75.3(39.2-204.3)$ & 0.083 \\
Vasopressors & 45 & $203.4(96.8-585.7)$ & 329 & $76.3(37.6-207.6)$ & $<0.0001$ \\
Renal replacement therapy & 37 & $203.4(104.2-565.0)$ & 337 & $78.8(37.6-209.3)$ & $<0.0001$ \\
Coma grade 3/4 & 152 & $110.2(40.1-279.6)$ & 213 & $75.0(37.6-207.6)$ & 0.15 \\
Late FABP1 (ng/mL; median(IQR)) & & & & & \\
Mechanical ventilation & 130 & $77.3(37.4-341.4)$ & 180 & $53.3(32.1-130.3)$ & 0.006 \\
Vasopressors & 58 & $116.4(53.8-502.10$ & 252 & $53.3(30.6-146.7)$ & $<0.0001$ \\
Renal replacement therapy & 30 & $112.0(51.6-448.3)$ & 280 & $33.3(159.7)$ & 0.053 \\
Coma grade 3/4 & 140 & $71.4(37.7-263.9)$ & 155 & $51.4(31.2-146.9)$ & 0.03 \\
\hline
\end{tabular}


This is a post-peer-review, pre-copyedit version of an article published in Dig Dis Sci. The final authenticated version is available online at: http://dx.doi.org/10.1007/s10620-020-06166-w".

\section{DISCUSSION}

\section{KEY RESULTS}

In this nested cohort study of 384 ALF patients, we report the first published analysis of FABP1 in a large series of wellcharacterized ALF patients due to AIH, HBV, and idiosyncratic DILI. Serum levels of FABP1 were significantly lower at late time points (days 3-5) in ALF TFS at day 21. However, after adjusting for significant covariates reflecting severity of illness (MELD), serum FABP1 (log) levels were not significantly associated with 21-day mortality measured either at early $(\mathrm{OR} 1.13, \mathrm{p}=0.29)$ or late $(\mathrm{OR} 1.27, \mathrm{p}=0.087)$ time points (Table 4).

Table 4 Overall. Early (day 1) and Late (days 3-5) predictors of 21-day mortality in 384 ALF patients

\begin{tabular}{|c|c|c|c|c|c|c|c|c|}
\hline \multirow[t]{2}{*}{ EARLY (Day 1-2) } & \multicolumn{4}{|c|}{ Unadjusted } & \multicolumn{4}{|c|}{$\begin{array}{c}\text { Multivariable Model } \\
(\mathrm{N}=339), \text { AUROC }=0.82\end{array}$} \\
\hline & $\mathrm{N}$ & OR & $95 \% \mathrm{Cl}$ & $P$-value & $\begin{array}{l}\text { Included in } \\
\text { Model }\end{array}$ & OR & $95 \% \mathrm{Cl}$ & $\mathrm{P}$-value \\
\hline $\log (F A B P 1)$ & 376 & 0.95 & $0.81-1.12$ & 0.56 & Yes & 1.13 & $0.90-1.40$ & 0.29 \\
\hline AlH & 384 & 1.19 & $0.64-2.22$ & \multirow{3}{*}{0.31} & Yes & 1.72 & $0.78-3.79$ & \multirow{3}{*}{0.27} \\
\hline DILI & 384 & 0.77 & $0.43-1.36$ & & Yes & 0.98 & $0.47-2.05$ & \\
\hline $\begin{array}{l}\text { Hepatitis B } \\
\text { (reference) }\end{array}$ & 384 & & & & Yes & & & \\
\hline White Blood Count & 376 & 1.04 & $0.99-1.08$ & 0.10 & No & & & \\
\hline Platelet Count & 375 & 1.00 & $1.00-1.00$ & 0.057 & Yes & 0.99 & $0.99-1.00$ & 0.0002 \\
\hline $\log ($ INR $)$ & 365 & 5.10 & $2.82-9.23$ & $<0.0001$ & Yes & 5.38 & $2.75-10.53$ & $<0.0001$ \\
\hline ALT & 367 & 1.00 & $1.00-1.00$ & 0.081 & No & & & \\
\hline Bilirubin & 369 & 1.07 & $1.04-1.10$ & $<0.0001$ & Yes & 1.10 & $1.06-1.14$ & $<0.0001$ \\
\hline Ammonia & 122 & 1.02 & $1.00-1.03$ & 0.0065 & No & & & \\
\hline Phosphate & 253 & 1.22 & $1.01-1.46$ & 0.040 & No & & & \\
\hline MELD & 353 & 1.09 & $1.06-1.13$ & $<0.0001$ & No & & & \\
\hline High Coma Grade & 373 & 3.50 & $1.98-6.18$ & $<0.0001$ & Yes & 3.97 & $2.01-7.85$ & $<0.0001$ \\
\hline $\begin{array}{l}\text { Mechanical } \\
\text { Ventilation }\end{array}$ & 382 & 4.68 & $2.44-8.98$ & $<0.0001$ & No & & & \\
\hline Vasopressors & 382 & 1.88 & $0.81-4.34$ & 0.14 & No & & & \\
\hline $\begin{array}{l}\text { Renal Replacement } \\
\text { Therapy }\end{array}$ & 382 & 2.03 & $0.77-5.37$ & 0.16 & No & & & \\
\hline \multirow[t]{2}{*}{ LATE (Day 3-7) } & \multicolumn{4}{|c|}{ Unadjusted } & \multicolumn{4}{|c|}{$\begin{array}{c}\text { Multivariable Model } \\
(\mathrm{N}=250), \text { AUROC }=0.76\end{array}$} \\
\hline & $\mathrm{N}$ & OR & $95 \% \mathrm{Cl}$ & P-value & $\begin{array}{l}\text { Included in } \\
\text { Model. }\end{array}$ & OR & $95 \% \mathrm{Cl}$ & P-value \\
\hline $\log (F A B P 1)$ & 315 & 128 & $1.03-1.58$ & 0.025 & Yes & 1.27 & $0.97-1.68$ & 0.087 \\
\hline AlH & 328 & 1.12 & $0.58-2.15$ & \multirow{3}{*}{0.58} & Yes & 1.17 & $0.52-2.65$ & \multirow{3}{*}{0.67} \\
\hline DILI & 328 & 0.81 & $0.44-1.51$ & & Yes & 0.82 & $0.37-1.84$ & \\
\hline $\begin{array}{l}\text { Hepatitis B } \\
\text { (reference) }\end{array}$ & 328 & & & & Yes & & & \\
\hline Platelet Count & 297 & 1.00 & $0.99-1.00$ & 0.0029 & Yes & 1.00 & $0.99-1.00$ & 0.021 \\
\hline INR & 289 & 2.34 & $1.67-3.30$ & $<0.0001$ & No & & & \\
\hline Bilirubin & 294 & 1.04 & $1.01-1.07$ & 0.0077 & No & & & \\
\hline Ammonia & 74 & 1.02 & $1.01-1.04$ & 0.010 & No & & & \\
\hline Lactate & 82 & 1.78 & $1.15-2.74$ & 0.0099 & No & & & \\
\hline MELD & 270 & 1.10 & $1.06-1.14$ & $<0.0001$ & Yes & 1.09 & $1.05-1.14$ & $<0.0001$ \\
\hline High Coma Grade & 308 & 5.21 & $2.75-9.86$ & $<0.0001$ & No & & & \\
\hline $\begin{array}{l}\text { Mechanical } \\
\text { Ventilation }\end{array}$ & 323 & 5.64 & $\begin{array}{l}2.90- \\
10.95\end{array}$ & $<0.0001$ & No & & & \\
\hline Vasopressors & 323 & 7.58 & $\begin{array}{l}2.30 \\
24.95\end{array}$ & 0.0009 & No & & & \\
\hline
\end{tabular}

\section{COMPARISON WITH LITERATURE}

ALF represents the severest form of hepatic injury resulting from massive necrosis. In the presence of hepatocyte injury, cytoplasmic proteins pass through large clefts embedded within a single endothelial cell layer and into the circulation. Hepatocyte damage results in FABP1 detection in blood [15] and has been proposed as a sensitive serum marker of hepatocellular damage in liver transplant recipients [16]. FABP1 has theoretical physiological properties that could

Karvellas, C. J.; Speiser, J. L.; Tremblay, M.; Lee, W. M.; Rose, C. F. \& Group, U. A. L. F. S. Elevated Serum Liver-Type Fatty Acid Binding Protein Levels in Non-acetaminophen Acute Liver Failure Patients with Organ Dysfunction. Digest Dis Sci, 2020 
This is a post-peer-review, pre-copyedit version of an article published in Dig Dis Sci. The final authenticated version is available online at: http://dx.doi.org/10.1007/s10620-020-06166-w".

potentially be advantageous as a prognostic biomarker. It is present in high abundance in hepatocytes (approximately 2.7 $\mathrm{mg} / \mathrm{g}$ of liver tissue), has a lower molecular mass (14 kDa) and shorter plasma half-life (11 min) compared to other cytosolic proteins (e.g., ALT) [17]. Due to its low molecular mass, FABP1 levels in the serum are influenced by both renal function and RRT. FABP1 is present in significantly smaller quantities in the kidney (proximal tubules) $[18,19]$.

FABP1 has previously been shown to be a diagnostic marker of liver injury in patients with DILI in the absence of ALF [20]. Recently, we evaluated the discriminatory ability of FABP1 in 198 acetaminophen (APAP)-ALF patients [8]. Elevated FABP1 levels were associated with increased mortality and improved prognostic discrimination when added to $\mathrm{KCC}$ and the ALFSG prognostic index [8]. In contrast, FABP1 did not appear to be as discriminatory between favorable (transplant-free survival) and unfavorable outcomes (death, requirement for LT) in other etiologies of ALF such as autoimmune, Hepatitis B, and DILI after adjusting for other covariates. One possible reason for this is that acetaminophen hepatotoxicity is characterized by hyperacute liver failure with the rapid (within 7 days usually) progression from onset of synthetic dysfunction to the development of ALF. Hence, there is likely a massive amount of hepatocyte necrosis occurring in a relatively short period of time [1]. In contrast to this, other causes of ALF such as autoimmune hepatitis, DILI, and Hepatitis B can follow a more subacute time course where the development of hepatocyte necrosis occurs over many days or weeks prior to the development of hepatic encephalopathy. This is further supported by a recent study by Eguchi and colleagues, which demonstrated median FABP1 levels of between 9 and $15 \mathrm{ng} / \mathrm{mL}$ in cirrhosis patients (i.e., minimal necrosis) with hepatocellular carcinoma, significantly lower that what were demonstrated in this study of non-APAP ALF [21].

FABP1 is involved in uptake, transport, and metabolism of long-chain fatty acids and has multiple biologic functions (fatty acid transport, storage, and metabolism) [22]. FABP1 also has an important role in facilitating hepatic fatty acid oxidation and is involved in trafficking and delivery of various ligands to cellular destinations such as enzymes, membranes, and nucleus [23]. FABP1 also acts as a cellular antioxidant as it contains a cysteine group and has been found to influence cell growth, since it has been demonstrated to be elevated during stages of mitosis [24]. Hepatic longchain fatty acid uptake is directly associated with FABP1 expression levels [25]. Therefore, an increased expression of hepatocyte FABP1 could be a compensatory mechanism in attempt to rescue energy-deprived hepatocytes undergoing necrosis. A failed attempt elicits necrosis, resulting in upregulated levels of FABP1 being released into circulation. Therefore, we speculate that while rapid massive necrosis of hepatocytes containing overexpressed levels of FABP1 could justify the association with non-spontaneous hepatic recovery in acetaminophen hepatotoxicity, due to the temporal relationship of hepatocyte necrosis in subacute liver failure (DILI, HBV, autoimmune), FABP1 was less discriminatory between favorable and unfavorable outcomes in these patient populations. This dichotomy is also reflected in ALF prognostic scores including the King's College Criteria (APAP vs. non-APAP) [12] and the ALFSG prognostic index (etiology subclasses) [3].

\section{LIMITATIONS}

The following limitations of this study warrant consideration. It is a nested case control study, and as such the event rate of the primary outcome (death or liver transplant at 21 days) was $75 \%$, higher than published in cohort series. Although patients were enrolled and samples were collected prospectively, analysis was done retrospectively and therefore can comment on association and discrimination (between transplant-free survivors and non-survivors/LT patients) and not on the absolute risks of death according to serum FABP1 levels. To account for potential confounding in the study design, we performed multivariable analysis to adjust for other significant covariates. The case-control design of the study may have introduced selection bias, as the primary outcome of transplant-free survival is automatically unbalanced within the clinical profile of the groups. However, in an attempt to reduce observation bias, data were collected prospectively and within this specific study design, researchers measuring FABP1 were blinded to the clinical and outcome data of patients at the time of patient selection and sample analysis. We did not have access to a control group with critical illness but not APAP ALF or acute-on-chronic liver failure as comparators; however, we did include a control group of healthy adults for comparison. Indeterminate/seronegative ALF patients were not included in this analysis due to ongoing causality reclassification at the time of sample pull. Further studies evaluating other markers related to the FABP pathway would be of value; however, we were unable to perform due to limitations in sample volume. Nonetheless despite these limitations, we believe these results

Karvellas, C. J.; Speiser, J. L.; Tremblay, M.; Lee, W. M.; Rose, C. F. \& Group, U. A. L. F. S. Elevated Serum Liver-Type Fatty Acid Binding Protein Levels in Non-acetaminophen Acute Liver Failure Patients with Organ Dysfunction. Digest Dis Sci, 2020 
This is a post-peer-review, pre-copyedit version of an article published in Dig Dis Sci. The final authenticated version is available online at: http://dx.doi.org/10.1007/s10620-020-06166-w".

are robust as they include 384 ALF cases from across 16 tertiary liver transplant centers comprising the US ALFSG. Furthermore, this builds on the body of evidence that suggests the differences in the natural history of hepatocyte injury in hyperacute liver failure (acetaminophen toxicity), acute/subacute liver failure (this study), and cirrhosis reflected by levels of FABP1.

\section{CONCLUSION}

In this first report of FABP1 in non-APAP ALF, FABP1 levels at late time points (day 3-5) were significantly lower in ALF patients who were alive without transplant at day 21 but not after adjusting for other covariates. Higher FABP1 levels were associated with the presence of increased organ failure.

Acknowledgment Members and institutions participating in the Acute Liver Failure Study Group 1998-2018 are as follows: W.M. Lee, M.D. (Principal Investigator); Anne M. Larson, M.D., Iris Liou, M.D., University of Washington, Seattle, WA; Oren Fix, M.D., Swedish Medical Center, Seattle, WA; Michael Schilsky, M.D., Yale University, New Haven, CT; Timothy McCashland, M.D., University of Nebraska, Omaha, NE; J. Eileen Hay, M.B.B.S., Mayo Clinic, Rochester, MN; Natalie Murray, M.D., Baylor University Medical Center, Dallas, TX; A. Obaid S. Shaikh, M.D., University of Pittsburgh, Pittsburgh, PA; Andres Blei, M.D., Northwestern University, Chicago, IL (deceased), Daniel Ganger, M.D., Northwestern University, Chicago, IL; Atif Zaman, M.D., University of Oregon, Portland, OR; Steven H.B. Han, M.D., University of California, Los Angeles, CA; Robert Fontana, M.D., University of Michigan, Ann Arbor, MI; Brendan McGuire, M.D., University of Alabama, Birmingham, AL; Raymond T. Chung, M.D., Massachusetts General Hospital, Boston, MA; Alastair Smith, M.B., Ch.B., Duke University Medical Center, Durham, NC; Robert Brown, M.D., Cornell/Columbia University, New York, NY; Jeffrey Crippin, M.D., Washington University, St Louis, MO; Edwin Harrison, Mayo Clinic, Scottsdale, AZ; Adrian Reuben, M.B.B.S., Medical University of South Carolina, Charleston, SC; Santiago Munoz, M.D., Albert Einstein Medical Center, Philadelphia, PA; Rajender Reddy, M.D., University of Pennsylvania, Philadelphia, PA; R. Todd Stravitz, M.D., Virginia Commonwealth University, Richmond, VA; Lorenzo Rossaro, M.D., University of California Davis, Sacramento, CA; Raj Satyanarayana, M.D., Mayo Clinic, Jacksonville, FL; and Tarek Hassanein, M.D., University of California, San Diego, CA; Constantine J. Karvellas MD, University of Alberta, Edmonton, AB; Jodi Olson MD, University of Kansas, Kansas City, KA; Ram Subramanian MD, Emory, Atlanta, GA; James Hanje MD, Ohio State University, Columbus, OH; Bilal Hameed MD, University of California San Francisco, CA. The University of Texas Southwestern Administrative Group included Grace Samuel, Ezmina Lalani, Carla Pezzia, and Corron Sanders, Ph.D., Nahid Attar, Linda S. Hynan, Ph.D., and the Medical University of South Carolina Data Coordination Unit included Valerie Durkalski, Ph.D., Wenle Zhao, Ph.D., Jaime Speiser, Catherine Dillon, Holly Battenhouse, and Michelle Gottfried. Special thanks to Dr. Elaine Leslie for study advice. Dr. Karvellas and Dr Rose are members of CDTRP Theme 3: Engineer and allocate a better graft. Author's contribution CJK conceived the study concept and design, performed analysis and interpretation of the data, and drafted the final manuscript. JLS performed statistical analysis and interpretation of data and critically revised the final manuscript. MT performed laboratory analysis and revised the final manuscript. WML, supervisor of entire US Acute Liver Failure Study Group (U-01 Grant), critically revised the manuscript for important intellectual content. CFR conceived the idea of the study, assisted in developing study design and interpretation of data, and critically revised the final manuscript for important intellectual content.

Funding The study was sponsored by NIH Grant U-01 58369 (from NIDDK) and the CDTRP ATIF Research Innovation Grant jointly supported by the Alberta Ministry of Economic Development and Trade, the University Hospital Foundation, Astellas Pharma Canada, and the Canadian Donation and Transplant Research Program. Compliance with Ethical Standards Conflict of interest All authors have no personal or funding conflict of interest.

\section{REFERENCES}

1’Grady JG, Williams R. Classifcation of acute liver failure. Lancet. 1993;342:743.

Karvellas, C. J.; Speiser, J. L.; Tremblay, M.; Lee, W. M.; Rose, C. F. \& Group, U. A. L. F. S. Elevated Serum Liver-Type Fatty Acid Binding Protein Levels in Non-acetaminophen Acute Liver Failure Patients with Organ Dysfunction. Digest Dis Sci, 2020 
This is a post-peer-review, pre-copyedit version of an article published in Dig Dis Sci. The final authenticated version is available online at: http://dx.doi.org/10.1007/s10620-020-06166-w".

2.McPhail MJ, Wendon JA, Bernal W. Meta-analysis of perfor-mance of Kings's College Hospital Criteria in prediction of out-come in non-paracetamol-induced acute liver failure. J Hepatol. 2010;53:492-499.

3.Koch DG, Tillman H, Durkalski V, Lee WM, Reuben A. Development of a model to predict transplant-free survival of patients with acute liver failure. Clin Gastroenterol Hepatol. 2016;14:1199-1206.

4.Karvellas CJ, Safnia N, Auzinger G, et al. Medical and psy-chiatric outcomes for patients transplanted for acetaminopheninduced acute liver failure: a case-control study. Liver Int Of J Int Assoc Study Liver. 2010;30:826-833.

5.Schmidt LE, Larsen FS. MELD score as a predictor of liver failure and death in patients with acetaminophen-induced liver injury. Hepatology. 2007;45:789-796.

6.Pelsers M, Hermens W, Glatz JF. Fatty acid-binding pro-teins as plasma markers of tissue injury. Clin Chim Acta. 2005;352:15-35.

7.Bass NM, Barker ME, Manning JA, Jones AL, Ockner RK. Aci-nar heterogeneity of fatty acid binding protein expression in the livers of male, female and clofbrate-treated rats. Hepatology. 1989;9:12-21.

8.Karvellas CJ, Speiser JL, Tremblay M, Lee WM, Rose CF, Group USALFS. Elevated FABP1 serum levels are associated with poorer survival in acetaminophen-induced acute liver fail-ure. Hepatology. 2017;65:938-949.

9.vonElm E, Altman DG, Egger M, Pocock SJ, Gotzsche PC, Vandenbroucke JP. Strengthening the reporting of observational studies in epidemiology (STROBE) statement: guidelines for reporting observational studies. BMJ. 2007;335:806-808.

10.Conn HO, Lieberthal MM, eds. The hepatic coma syndromes and lactulose. Philadelphia: Williams \& Wilkins; 1979.

11.Atterbury CE, Maddrey WC, Conn HO. Neomycin-sorbitol and lactulose in the treatment of acute portal-systemic encephalop-athy: a controlled, double-blind clinical trial. Am J Dig Dis. 1978;23:398-406.

12.O'Grady JG, Alexander GJ, Hayllar KM, Williams R. Early indicators of prognosis in fulminant hepatic failure. Gastroen-terology. 1989;97:439-445.

13.Kamath PS, Wiesner RH, Malinchoc M, et al. A model to pre-dict survival in patients with end-stage liver disease. Hepatol-ogy. 2001;33:464-470.

14.Li X, Song X, Gray RH. Comparison of the missing-indicator method and conditional logistic regression in 1:m matched case-control studies with missing exposure values. Am J Epidemiol. 2004;159:603-610.

15.van den Broek MA, Bloemen JG, Dello SA, van de Poll MC, Olde Damink SW, Dejong CH. Randomized controlled trial ana-lyzing the efect of 15 or 30 min intermittent Pringle maneu-ver on hepatocellular damage during liver surgery. J Hepatol. 2011;55:337-345.

16.Pelsers MM, Morovat A, Alexander GJ, Hermens WT, Trull AK, Glatz JF. Liver fatty acid-binding protein as a sensitive serum marker of acute hepatocellular damage in liver transplant recipients. Clin Chem. 2002;48:2055-2057.

17.Vergani L, Fanin M, Martinuzzi A, et al. Liver fatty acid-bind-ing protein in two cases of human lipid storage. Mol Cell Bio-chem. 1990;98:225-230.

18. Negishi K, Noiri E, Doi K, et al. Monitoring of urinary L-type fatty acid-binding protein predicts histological severity of acute kidney injury. Am J Pathol. 2009;174:1154-1159.

19.Pelsers MM, Namiot Z, Kisielewski W, et al. Intestinal-type and liver-type fatty acid-binding protein in the intestine: tissue distri-bution and clinical utility. Clin Biochem. 2003;36:529-535.

Karvellas, C. J.; Speiser, J. L.; Tremblay, M.; Lee, W. M.; Rose, C. F. \& Group, U. A. L. F. S. Elevated Serum Liver-Type Fatty Acid Binding Protein Levels in Non-acetaminophen Acute Liver Failure Patients with Organ Dysfunction. Digest Dis Sci, 2020 
20.Mikus M, Drobin K, Gry M, et al. Elevated levels of circulating CDH5 and FABP1 in association with human druginduced liver injury. Liver Int Of J Int Assoc Study Liver. 2016;37:132-140.

21.Eguchi A, Hasegawa H, Iwasa M, et al. Serum liver-type fatty acid-binding protein is a possible prognostic factor in human chronic liver diseases from chronic hepatitis to liver cirrhosis and hepatocellular carcinoma. Hepatol Commun. 2019;1:1-11.

22.Bass NM. The cellular fatty acid binding proteins: aspects of struc-ture, regulation, and function. Int Rev Cytol. 1988;111:143-184.23. Veerkamp JH, van Moerkerk HT. Fatty acid-binding pro-tein and its relation to fatty acid oxidation. Mol Cell Biochem. 1993;123:101-106.

24.Custer RP, Sorof S. Mitosis in hepatocytes is generally associated with elevated levels of the target polypeptide of a liver carcinogen. Diferentiation. 1985;30:176-181.

25.Wang G, Chen QM, Minuk GY, Gong Y, Burczynski FJ. Enhanced expression of cytosolic fatty acid binding protein and fatty acid uptake during liver regeneration in rats. Mol Cell Biochem. 2004;262:41-49. 